\title{
C-reactive protein may misdiagnose prosthetic joint infections, particularly chronic and low-grade infections
}

Daniel Pérez-Prieto, $1,2{ }^{*}$

Phone +34-932483196

Emaildperezprieto@parcdesalutmar.cat

María E. Portillo, 3

Lluís Puig-Verdié, 1

Albert Alier, 1

Santos Martínez, 1

Lluisa Sorlí, 4

Juan P. Horcajada, 4

Joan C. Monllau, 1,2

1 Orthopaedic Department, Hospital del Mar - Universitat Autònoma de Barcelona, Barcelona, Spain

2 Department of Orthopaedic Surgery and Traumatology, Hospital Quiron-Dexeus, Barcelona, Spain

3 Microbiology Department, Hospital de Navarra, Pamplona, Spain

4 Infectious Diseases Department, Hospital del Mar, Barcelona, Spain

\section{Abstract \\ Background}

Periprosthetic tissue cultures, sonication and synovial fluid cultures remain 
the gold standard for prosthetic joint infection (PJI) diagnosis. However, some $15-20 \%$ culture-negative PJI are still reported. Therefore, there is the need for other diagnostic criteria. One point of concern relative to the different definitions of PJI is as to the inclusion of the c-reactive protein (CRP) and the erythrocyte sedimentation rate (ESR) as diagnostic criteria for PJI despite them being non-specific inflammatory blood tests.

\section{Purpose}

The purpose of the present study was to determine the relevance of CRP and the ESR in the diagnosis of PJI.

\section{Methods}

All PJI with positive cultures over a two-year period in two hospitals were reviewed. The main variables of the present study were the type of prosthesis and the CRP level. More information was recorded in those patients with normal CRP: radiographs, physical examination records and the ESR.

\section{Results}

Seventy-three patients were included in study. Preoperative CRP levels were normal (lower than $0.8 \mathrm{mg} / \mathrm{dl}$ ) in 23 patients, representing $32 \%$ of all PJI with positive cultures. Low virulence microorganisms, 12 coagulasenegative staphylococci and four $P$. acnes, grew in most of them. They represented $70 \%$ of all PJI with normal CRP levels. In addition, 17 patients (23\% of all PJI with positive cultures) had a normal ESR, a normal physical examination (they only presented with pain) and no clear loosening was observed in the radiographs.

\section{Conclusions}

Per the American Association of Orthopaedic Surgeons (AAOS) guidelines or the Musculoskeletal Infection Society (MSIS), 23\% of the patients in the present study with PJI would never have been identified. Blood inflammatory markers such as the CRP level and ESR may not be accurate as diagnostic tools in PJI, particularly to identify low-grade and 
chronic PJI.

\section{Keywords}

\section{Introduction}

Prosthetic joint infection (PJI) is a major complication after arthroplasty. The rates of PJI vary depending on the joint affected. It ranges from $5 \%$ in total knee arthroplasty (TKA) to $2 \%$ in total hip arthroplasty (THA) or reverse shoulder arthroplasty (RSA) [1].

However, these rates may be even higher as some 15 to $20 \%$ culture negative PJI are still being reported despite the recently developed microbiological methods for the diagnosis of PJI [1, 2, 3]. In those culturenegative PJI, some other non-microbiological diagnostic tools are crucial to detecting and treating the infection. Moreover, microbiological diagnostic is usually available several days after surgery. Therefore, there is the need for preoperative diagnosis [2]. For that purpose, physical examination (e.g. sinus tract [1]), joint aspiration (e.g. synovial fluid culture, leukocyte count [3]) or blood markers (e.g. c-reactive protein [CRP] and erythrocyte sedimentation rate [ESR] [4]) have been included as diagnostic criteria in several definitions of PJI. The most well-known are the Infectious Diseases Society of America (IDSA)/Zimmerli criteria [5,6] (the most used in Europe $[2,3,7,8,9,10])$ and the American Association of Orthopaedic Surgeons (AAOS)/ Musculoskeletal Infection Society (MSIS) criteria [11, 12] (the most used in the United States $[13,14,15])$. One point of concern is the inclusion of CRP and ESR in the diagnostic criteria for PJI. Whereas the MSIS guidelines include the two markers as one criterion to diagnose PJI [11], the AAOS guidelines conclude that infection is unlikely and there is no need for more tests [12] in cases with normal CRP level and ESR. The Zimmerli definition of PJI does not even mention ESR and PCR [5].

The ESR and CRP level are non-specific inflammatory markers and thus can be elevated in conditions other than PJI [16]. In addition, and more importantly, those markers may be normal in the so-called low-grade PJI. Therefore, they may lead to a misdiagnosis based on some of the 
aforementioned criteria [17].

The purpose of the present study is to determine the relevance of CRP and the ESR in the diagnosis of PJI. The hypothesis is that the CRP level and ESR are of little value as diagnostic criterion for PJI.

\section{Material and methods}

A retrospective analysis of all PJI diagnosed in two university hospitals over two years was performed. PJI was defined in accordance with the Zimmerli criteria [5] (Table 1).

\section{Table 1}

Zimmerli criteria for $\mathrm{PJI}^{\mathrm{a}}$

1. Presence of a sinus tract communicating with the prosthetic joint

2. Presence of purulence without another known aetiology surrounding the prosthetic device

3. Acute inflammation consistent with infection on histopathological examination of periprosthetic tissue

4. $>2,000 / \mathrm{mm}^{3}$ leukocytes in the synovial fluid and/or $>65 \%$ of neutrophils

5. Growth of identical microorganisms in at least two intraoperative cultures or a combination of preoperative aspiration and intraoperative cultures in the case of a microorganism of low virulence (e.g. coagulase-negative staphylococci, Propionibacterium acnes). In the case of a virulent microorganism (e.g. Staphylococcus aureus, Escherichia coli), growth in a single specimen from synovial fluid, periprosthetic tissue and/or sonication fluid may also represent PJI.

${ }^{a}$ Only one of the five criteria is required for the diagnosis of PJI

Demographic data such as age, gender and comorbidities were collected.

The main variables of the present study were the type of prosthesis and the CRP level. More information was recorded in those patients with normal CRP level. That information included radiographs, physical examination records and the ESR. The type of infection was also registered (acute postoperative, acute hematogenous or chronic PJI) [5].

Per the local laboratory, the CRP level was considered normal if the value was under $0.8 \mathrm{mg} / \mathrm{dl}$. For ESR, the cut-off provided by the laboratory was $34 \mathrm{~mm} / \mathrm{h}$. 
To give more strength to the study, patients with rheumatic diseases and culture-negative PJI were excluded.

Cultures were defined as positive when there was microorganism growth in preoperative aspirate, periprosthetic tissue cultures or sonication fluid cultures. It was considered a positive sonication fluid culture when the same organism grew 50 or more colony-forming units (CFU)/mL $[18,19]$. However, any growth in the sonication fluid culture was considered positive when the patient had previously received antibiotics [8]. In that sense, preoperative prophylaxis was not considered as it has been shown to have no influence on cultures [20]. Low-virulence microorganisms such as coagulase-negative staphylococci (CNS), Corynebacterium species, Bacillus species, or Propionibacterium species were considered pathogens if the same organism was isolated in at least two samples or in one sample if at least one clinical criterion for PJI was also present $[5,6]$.

The results were expressed as a mean and standard deviation (SD) or gross value and percentage.

\section{Results}

A total of 2,236 joint replacements were performed in the hospitals. There were $92 \mathrm{PJI}$, representing an infection rate of $4 \%$. Five patients suffering from rheumatic diseases were excluded. Nine patients who had culture negative PJI were also excluded. It was not possible to obtain preoperative CRP levels in two patients (one TKA and one RSA) and the ESR was not available in three patients (two THA and one TKA). In the end, 73 patients were included for further study.

The mean age was 69 years old (SD, eight years) and there were 33 males (45\%). A flow chart can be seen in Fig. 1.

\section{Fig. 1}

Flow-chart of patients assessed in the present study. $P J I$ prosthetic joint infection, $C R P$ c-reactive protein, $E S R$ erythrocyte sedimentation rate, $P E$ physical examination. According to AAOS guidelines [12] those patients would have been classified as aseptic cases with no further study 


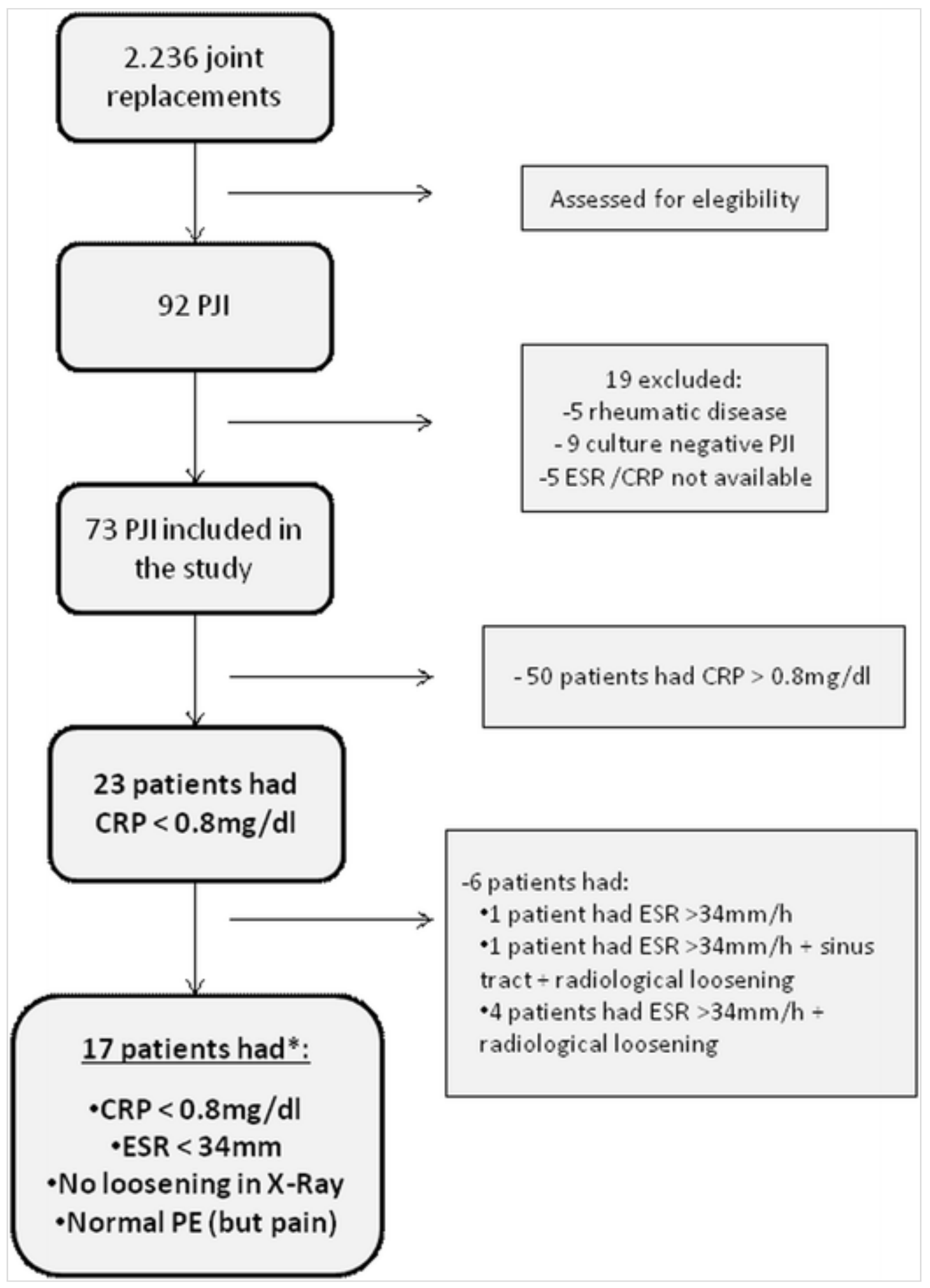

There were 30 TKA infections that represented $41 \%$ of the sample and 29 hip arthroplasties that corresponded to $40 \%$ of all PJI cases. The rest were 13 shoulder arthroplasties (18\%) and one total elbow arthroplasty (TEA) that corresponded to $1 \%$ of the study group.

Preoperative CRP levels were normal (lower than $0.8 \mathrm{mg} / \mathrm{dl}$ ) in 23 patients, representing $32 \%$ of all PJI with positive cultures.

Low virulence microorganisms, $12 \mathrm{CNS}$ and four $P$. acnes, grew in most of 
them. They represented $70 \%$ of all PJI with normal CRP levels. The detailed data on the type of prosthesis and microorganism can be seen in Table 2 .

Table 2

Type of prosthesis and microorganism of patients with normal CRP

\begin{tabular}{|c|c|c|c|c|c|}
\hline Microorganism & TKA & THA & $\begin{array}{l}\text { Shoulder } \\
\text { arthroplasty }\end{array}$ & $\begin{array}{l}\text { Total elbow } \\
\text { arthroplasty }\end{array}$ & Total \\
\hline CNS & 1 & 7 & 3 (RSA) & 1 & 12 \\
\hline S. aureus & 1 & 2 & & & 3 \\
\hline P. acnes & & 3 & $1(\mathrm{~S}-\mathrm{HA})$ & & 4 \\
\hline $\begin{array}{l}\text { Gram negative } \\
\text { bacteria }\end{array}$ & & 1 & $1(\mathrm{~S}-\mathrm{HA})$ & & 2 \\
\hline S. dysgalactiae & & & 1 (RSA) & & 1 \\
\hline C. albicans & & 1 & & & 1 \\
\hline Total & 2 & 14 & 6 & 1 & 23 \\
\hline
\end{tabular}

When those 23 patients with normal CRP levels were further analyzed, it was found that 17 patients (23\% of all PJI with positive cultures) had a normal ESR, a normal physical examination (they only presented with pain) and no clear loosening was observed in the radiographs. All those 17 patients had chronic infections. More information about these patients can be seen in Fig. 1 .

\section{Discussion}

The main result of the present study is that the CRP level misdiagnoses PJI, particularly chronic and low-grade infections. When CRP and the ESR are combined, they still misdiagnose PJI. In that sense, the hypothesis has been confirmed.

For many years the ESR and mostly the CRP level have been linked to a diagnosis of PJI [4]. They are extensively used worldwide. However, they are non-specific blood inflammatory markers [17]. Moreover, it is important to keep the pathogenesis of PJI in mind as bacteria can live dormant beneath 
the biofilm and not interact with inflammatory cells. This occurs in most chronic infections $[1,17]$. This fact may explain why the CRP level and ESR are normal in $23 \%$ of the study patients. It is worth mentioning that those patients in this study presented with only pain and no other symptoms. Therefore, those patients would have been misdiagnosed according to AAOS guidelines [12]. None of the patients with a normal CRP level in the present study had an acute PJI. One might think that the level of CRP would be useful in the diagnosis of acute PJI. However, acute infections are evident upon physical examination (drainage, fever, redness, etc.) and joint aspiration (pus or leukocyte count), so no further non-specific blood marker like the CRP is needed [5]. Moreover the CRP level rises after surgery even if there is no infection, making it is very unreliable [21].

When it comes to the type of microorganism, few studies have found that low-grade PJI (those related to bacteria such as P. acnes or CNS) can occur with normal serum CRP [22] (and more recently the same has been found to be true of synovial fluid) [21]. Similar results have been found in the present study as $70 \%$ of PJI in patients with a normal CRP level were due to those microorganisms. However, it is relevant that $30 \%$ of patients with normal CRP had a PJI caused by virulent bacteria (for instance, S. aureus), which is being reported for the first time here.

CRP levels have also been analyzed depending on the type of the prosthesis that was infected. Some authors have found that shoulder PJI show a substantial number of normal CRP levels [16]. It may be explained in part by the high prevalence of $P$. acnes in shoulder infections [23]. They have recommended using a different cut-off for CRP depending on the prosthesis that the surgeon is dealing with [16]. In the present study, a high percentage of shoulder arthroplasties also presented with normal CRP levels even though there was an underlying infection. Conversely, there were also TKA and total hip arthroplasties (THA) in this study so using different cut-offs would not have been a solution.

Recently, some of the authors of the AAOS/MSIS guidelines have concluded that their criteria may not be as accurate as they had thought as they state that "infection may be present even if the patient does not meet these criteria" [24, 25]. Moreover, they have found that one third of the patients with confirmed PJI in one study did not meet the MSIS criteria [24]. In a similar way, Frangiamore et al. found that MSIS criteria have low sensitivity 
to ruling out PJI [26]. This is especially important in the case of the level of $\mathrm{CRP}$ as has been found in the present study.

Future studies should evaluate whether diagnostic criteria that do not include the level of CRP and the ESR are more reliable compared with those that do include them.

Several limitations can be found in the present study. The most important is its retrospective design. Another limitation is the small sample size. Additionally, there were five patients in which the CRP level and the ESR were not available. One minor limitation is that all types of infection (acute and chronic) have been included in the present study. However, no acute infection had a normal CRP level in the present study.

\section{Conclusions}

Blood inflammatory markers such as the CRP level and ESR may be of little value as diagnostic tools in PJI, particularly to identify most low-grade and chronic PJI. Diagnostic criteria that use ESR and CRP might misdiagnose up to one fourth of PJI.

\section{Acknowledgements}

We are grateful to Mr. Eric L Goode for his help with English correction and style.

Compliance with ethical standards

Conflict of interest All authors declare that they do not have any conflict of interest.

Funding There is no funding source.

Ethical approval This is a retrospective observational study without intervention in humans or animals performed by any of the authors.

\section{References}

1. Zimmerli W, Trampuz A, Ochsner PE (2004) Prosthetic-joint infections. N Engl J Med 351:1645-1654. doi: 10.1056/NEJMra040181 
2. Portillo ME, Salvado M, Trampuz A et al (2015) Improved diagnosis of orthopedic implant-associated infection by inoculation of sonication fluid into blood culture bottles. J Clin Microbiol 53:1622-1627. doi: 10.1128/JCM.03683-14

3. Trampuz A, Hanssen AD, Osmon DR et al (2004) Synovial fluid leukocyte count and differential for the diagnosis of prosthetic knee infection. Am J Med 117:556-562. doi: 10.1016/j.amjmed.2004.06.022

4. Berbari E, Mabry T, Tsaras G et al (2010) Inflammatory blood laboratory levels as markers of prosthetic joint infection: a systematic review and meta-analysis. J Bone Joint Surg Am 92:2102-2109. doi: 10.2106/JBJS.I.01199

5. Zimmerli W (2014) Clinical presentation and treatment of orthopaedic implant-associated infection. J Intern Med 276:111-119. doi: 10.1111/joim. 12233

6. Osmon DR, Berbari EF, Berendt AR et al (2013) Executive summary: diagnosis and management of prosthetic joint infection: clinical practice guidelines by the infectious diseases society of America. Clin Infect Dis 56:1-10. doi: $10.1093 / \mathrm{cid} / \mathrm{cis} 966$

7. Minassian AM, Newnham R, Kalimeris E et al (2014) Use of an automated blood culture system (BD BACTEC) for diagnosis of prosthetic joint infections: easy and fast. BMC Infect Dis 14:233. doi: 10.1186/1471-2334-14-233

8. Portillo ME, Salvado M, Alier A et al (2014) Advantages of sonication fluid culture for the diagnosis of prosthetic joint infection. J Infect 69:3541. doi: $10.1016 /$ j.jinf.2014.03.002

9. Puig-Verdie L, Alentorn-Geli E, Gonzalez-Cuevas A et al (2013) Implant sonication increases the diagnostic accuracy of infection in patients with delayed, but not early, orthopaedic implant failure. Bone Joint J 95-B:244-249. doi: 10.1302/0301-620X.95B2.30486

10. Borens O, Corvec S, Trampuz A (2012) Diagnosis of periprosthetic joint infections. Hip Int 22(Supp1 8):S9-S14. doi: 
11. Parvizi J, Zmistowski B, Berbari EF et al (2011) New definition for periprosthetic joint infection: from the workgroup of the musculoskeletal infection society. Clin Orthop Relat Res 469:2992-2994. doi:

10.1007/s11999-011-2102-9

12. Della Valle C, Parvizi J, Bauer TW et al (2011) American Academy of Orthopaedic Surgeons clinical practice guideline on: the diagnosis of periprosthetic joint infections of the hip and knee. J Bone Joint Surg Am 93:1355-1357. doi: 10.2106/JBJS.9314ebo

13. Ahmad SS, Shaker A, Saffarini M et al (2016) Accuracy of diagnostic tests for prosthetic joint infection: a systematic review. Knee Surg Sports Traumatol Arthrosc. doi: 10.1007/s00167-016-4230-y

14. Alijanipour P, Bakhshi H, Parvizi J (2013) Diagnosis of periprosthetic joint infection: the threshold for serological markers. Clin Orthop Relat Res 471:3186-3195. doi: 10.1007/s11999-013-3070-z

15. Matsen Ko L, Parvizi J (2016) Diagnosis of periprosthetic infection: novel developments. Orthop Clin N Am 47:1-9. doi:

10.1016/j.ocl.2015.08.003

16. Piper KE, Fernandez-Sampedro M, Steckelberg KE et al (2010) Creactive protein, erythrocyte sedimentation rate and orthopedic implant infection. PLoS One 5:e9358. doi: 10.1371/journal.pone.0009358

17. Renz N, Muller M, Perka C, Trampuz A (2016) Implant-associated infections - diagnostics. Chirurg. doi: 10.1007/s00104-016-0234-x

18. Renz N, Cabric S, Janz V, Trampuz A (2015) Sonication in the diagnosis of periprosthetic infections: significance and practical implementation. Orthopade 44:942-945. doi: 10.1007/s00132-015-3192-y

19. Trampuz A, Piper KE, Jacobson MJ et al (2007) Sonication of removed hip and knee prostheses for diagnosis of infection. N Engl J Med 357:654-663. doi: 10.1056/NEJMoa061588 
20. Perez-Prieto D, Portillo ME, Puig-Verdie L et al (2016) Preoperative antibiotic prophylaxis in prosthetic joint infections: not a concern for intraoperative cultures. Diagn Microbiol Infect Dis 86:442-445. doi: 10.1016/j.diagmicrobio.2016.09.014

21. Deirmengian CA, Citrano PA, Gulati S et al (2016) The c-reactive protein may not detect infections caused by less-virulent organisms. J Arthroplasty 31(9 Suppl):152-155. doi: 10.1016/j.arth.2016.01.060

22. McArthur BA, Abdel MP, Taunton MJ et al (2015) Seronegative infections in hip and knee arthroplasty: periprosthetic infections with normal erythrocyte sedimentation rate and c-reactive protein level. Bone Joint J 97-B:939-944

23. Portillo ME, Corvec S, Borens O, Trampuz A (2013)

Propionibacterium acnes: an underestimated pathogen in implantassociated infections. Biomed Res Int 2013:804391. doi: $10.1155 / 2013 / 804391$

24. Koh IJ, Cho W-S, Choi NY et al (2015) How accurate are orthopedic surgeons in diagnosing periprosthetic joint infection after total knee arthroplasty?: A multicenter study. Knee 22:180-185. doi: 10.1016/j.knee.2015.02.004

25. Parvizi J, Gehrke T, Chen AF (2013) Proceedings of the international consensus on periprosthetic joint infection. Bone Joint J 95B:1450-1452. doi: 10.1302/0301-620X.95B11.33135

26. Frangiamore SJ, Siqueira MBP, Saleh A et al (2016) Synovial cytokines and the MSIS criteria are not useful for determining infection resolution after periprosthetic joint infection explantation. Clin Orthop Relat Res 474:1630-1639. doi: 10.1007/s11999-016-4710-x 